\title{
The origin of the compressibility anomaly in amorphous silica: a molecular dynamics study
}

\author{
Andrew M Walker ${ }^{1}$, Lucy A Sullivan ${ }^{1}$, Kostya Trachenko ${ }^{1}$, \\ Richard P Bruin ${ }^{1}$, Toby O H White ${ }^{1}$, Martin T Dove ${ }^{1,4}$, \\ Richard P Tyer ${ }^{2}$, Ilian T Todorov ${ }^{2}$ and Stephen A Wells ${ }^{3}$ \\ ${ }^{1}$ Department of Earth Sciences, University of Cambridge, Downing Street, \\ Cambridge CB2 3EQ, UK \\ ${ }^{2}$ CCLRC Daresbury Laboratory, Daresbury, Warrington, Cheshire WA4 4AD, UK \\ ${ }^{3}$ Center for Biological Physics, Arizona State University, Bateman Physical Sciences Building, \\ Tempe, AZ 85287-1504, USA \\ E-mail: mtd10@cam.ac.uk
}

Received 23 February 2007, in final form 4 April 2007

Published 1 June 2007

Online at stacks.iop.org/JPhysCM/19/275210

\begin{abstract}
We propose an explanation for the anomalous compressibility maximum in amorphous silica based on rigidity arguments. The model considers the fact that a network structure will be rigidly compressed in the high-pressure limit, and rigidly taut in the negative pressure limit, but flexible and hence softer at intermediate pressures. We validate the plausibility of this explanation by the analysis of molecular dynamics simulations. In fact this model is quite general, and will apply to any network solid, crystalline or amorphous; there are experimental indications that support this prediction. In contrast to other ideas concerning the compressibility maximum in amorphous silica, the model presented here does not invoke the existence of polyamorphic phase transitions in the glass phase.
\end{abstract}

\section{Introduction}

Most materials get stiffer under pressure, due to the increased compaction of the constituent atoms. It is therefore interesting that some materials, including amorphous silica, actually get softer on compression; in the case of amorphous silica, there is a maximum in compressibility from experiment at a pressure of around $2 \mathrm{GPa}$ [1]. This phenomenon remains unexplained. In this paper we use molecular dynamics simulations to confirm a surprisingly simple hypothesis concerning the origin of the compressibility maximum in amorphous silica, that the origin of the compressibility maximum arises from the flexibility of the network at around $2 \mathrm{GPa}$, compared to when it is compacted at high pressure or extended at low pressure. Unlike previous

4 Author to whom any correspondence should be addressed. 
interpretations based on properties of the amorphous state (discussed below) the flexibility hypothesis is equally valid for crystalline networks, such as zeolites and other silicates, as well as amorphous solids, and we believe will have wide applicability to studies of many classes of materials at high pressure.

The hypothesis presented in this paper rests on the fact that amorphous silica is primarily composed of an infinite network of corner-linked $\mathrm{SiO}_{4}$ tetrahedra. At the limit of high pressure, the tetrahedra are compacted together, and the forces are such that the tetrahedra will deform and eventually bonds will be broken and reformed [2]. These are high-energy processes, and hence the compressibility in this limit will be reduced. Now consider the opposite limit, namely a large negative pressure leading to an expansion of the material. In this limit, the network of tetrahedra will be stretched taut, and any changes in volume will require stretching of the covalent $\mathrm{Si}-\mathrm{O}$ bonds. Once again, this is a relatively high-energy process, and the compressibility will be reduced. In the intermediate pressure range (around ambient pressure) volume changes can be accommodated through buckling of the tetrahedral network without deformations of the tetrahedra. We know from previous simulation work [2-6] that at ambient pressure there is a high degree of network flexibility that is accessible to thermal fluctuations; these thermal fluctuations are called 'Floppy Modes' in the context of glasses or 'Rigid Unit Modes' (RUMs) in the context of crystalline materials. Buckling of the network when there is a high degree of inherent flexibility is a low-energy process, and hence the compressibility will be higher in this pressure range. These various extreme cases are illustrated in cartoon form in figure 1. A similar flexibility window has recently been discussed in the case of zeolites [7]. This simple comparison of two pressure extremes with the intermediate pressure suggests that the origin of the compressibility maximum arises from the existence of the network flexibility within an intermediate range of pressures. We call this the flexibility hypothesis.

The flexibility hypothesis contrasts with other suggestions as to the origin of the compressibility maximum. One is to assume that the maximum is a signature of a polyamorphic structural phase transition in the glass [8,9] or associated with other structural/topological changes within the atomic configuration. However, previous work using the models in this paper show clearly that there are no such changes over the pressure range of interest $[3,4]$. Anticipating the results presented below, we note that there is no correlation between the compressibility maximum and structural changes, allowing us to rule out polyamorphic phase transitions as the origin of the compressibility maximum. Another explanation is that the compressibility maximum is associated with the existence of two-level states [10]. In fact, we have previously seen these states in our simulation work $[5,6]$, and we will see them in the present study (see below), there is no evidence that they are a significant factor in the compressibility anomaly. In fact, we note that in neither of these two suggestions is there any developed theoretical working; these suggestions appear to be based on linking different factors that appear to be unique to the amorphous state.

In this paper we will use the molecular dynamics (MD) simulation method to probe the flexibility hypothesis for amorphous silica in a number of ways. We will first establish that the compressibility maximum can be reproduced using different atomic configurations and different interatomic potentials. The flexibility hypothesis proposes that the greatest effect of pressure on the structure should be on the buckling of the network at intermediate pressures, that expansion of the $\mathrm{Si}-\mathrm{O}$ bond should occur at larger negative pressures, and that distortions of the structure topology (bond breaking and changing coordination) should occur only at pressures significantly above the compressibility maximum. This last point has actually been established in our previous simulation work [2-4]. We provide evidence from analysis of bond lengths, contact distances, and their variances. The flexibility hypothesis also proposes that the network is most flexible at pressures around the compressibility maximum, and we demonstrate this 
a)

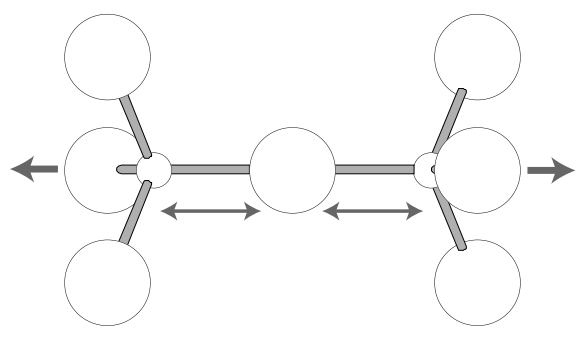

b)

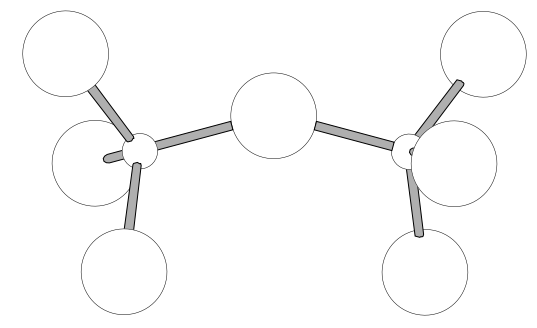

c)

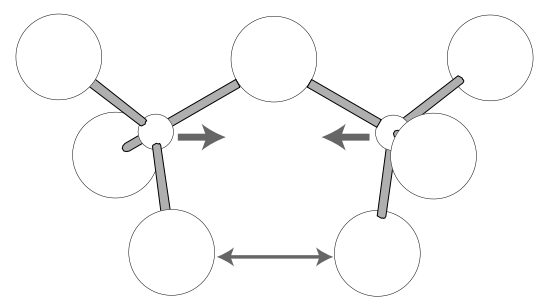

Figure 1. Cartoon representation of three configurations of a pair of linked $\mathrm{SiO}_{4}$ tetrahedra in amorphous silica; large spheres represent oxygen atoms and small spheres represent silicon atoms. (a) Fully stretched conformation, in which any further increase in the volume of the conformation can only be accomplished by stretching bonds and deforming the $\mathrm{SiO}_{4}$ tetrahedra-in practice pairs of tetrahedra will not be stretched to this limit. (b) The relaxed state, in which expansion of contraction of the volume occupied by the atoms can be accommodated easily by flexing of the central $\mathrm{O}-\mathrm{Si}-\mathrm{O}$ bond with no deformations of the $\mathrm{SiO}_{4}$ tetrahedra required. (c) The conformation under pressure, in which one or more oxygen atoms are forced to be close together. Under this state, the only way to reduce the volume of the conformation is to distort the $\mathrm{SiO}_{4}$ tetrahedra.

through analysis of tetrahedral thermal motions. It is the nature of such work that we cannot prove the flexibility hypothesis as the origin of the compressibility maximum in amorphous silica, but we believe that the supporting evidence from our simulations and the plausibility of the flexibility hypothesis form a compelling case. One aspect of the flexibility hypothesis is that it should be applicable to crystalline network structures, and we discuss this point in section 4 .

\section{Methods}

To explore the plausibility of the flexibility hypothesis as the origin of the compressibility maximum, we have performed molecular dynamics (MD) simulations on various defect-free configurations of amorphous silica produced using the Wooten-Winer-Weaire method [11, 12] as used in our previous simulation work $[2,3,5,6]$. One configuration contained $512 \mathrm{SiO}_{4}$ 
tetrahedra and two contained 4096 tetrahedra; the two larger configurations differ in that one does not contains any four-membered rings and the other does (labelled A and B respectively below). We used two different model interatomic potentials (labelled TTAM [13] and BKS [14] below).

The MD simulations were performed using the DL_POLY_3 package [15, 16]. Each MD simulation lasted $100 \mathrm{ps}$ and was performed at a temperature of $50 \mathrm{~K}$. The time step was $0.001 \mathrm{ps}$, and the velocity Verlet scheme [17] was used. Our MD runs were split into two segments, each of which began with 10000 equilibration steps where the velocities of the particles were rescaled every time step such that the system equilibrated to our chosen set temperature. The first 50000 steps sampled the isothermal isobaric (NPT) ensemble [18]; relaxation times of $1.0 \mathrm{ps}$ were used for the thermostat and barostat. We used this segment of the simulation to extract the relationship between pressure and volume for each combination of glass configuration and interatomic interaction model. Because the fluctuation in the cell volume was small compared to the difference in cell volume between different pressure points, we used the final configuration and cell volume from the first 50000 steps to begin the second portion of the MD run. This was performed using the microcanonical (NVE) ensemble, and used for analysis of the dynamics of the glass model. By using grid computing [19-22] and data management methods [21-23] we were able to run many more simulations than would be normal for this type of study. Pressures were varied between -5 and $+5 \mathrm{GPa}$. The set temperature was $50 \mathrm{~K}$. By monitoring the pair distribution functions and the topology, we demonstrated that there are no changes in structure with pressure that could be signatures of a polyamorphic phase transition, as per our previous work [2-4].

\section{Results}

\subsection{Volume and compressibility}

Volume versus pressure curves for the different configurations and interatomic potentials are shown in figure 2 (top). The curves differ negligibly with different configurations and sample size, but they are sensitive to the model interatomic potential. However, they all have the same characteristic shape with a maximum slope at intermediate pressures; this is the sign of a compressibility maximum. The curves were fitted by Chebyshev polynomials, and differentiated to give the compressibility curves shown in figure 2 (bottom). It can be seen that all models yield a maximum in compressibility - thus the existence of the maximum is not dependent on either the details of the atomic configuration or the form of the interatomic potentials. We note that the exact pressure of the maximum is not precisely identified by this approach because it is very sensitive to the polynomial fitting. It is this sensitivity that causes the main difference between the shapes of the different compressibility curves.

The experimental compressibility for amorphous silica is around $0.026 \mathrm{GPa}^{-1}$ at zero pressure, with the maximum value of around $0.033 \mathrm{GPa}^{-1}$ [1]. Our results (figure 2) are slightly larger than these data, with the discrepancy being larger for the TTAM potential than for the BKS potential. These discrepancies will arise from the details of the interatomic potentials.

\subsection{Interatomic distances}

Figure 3 (top) compares the near-neighbour distances and $V^{1 / 3}$ for the TTAM potential and the 4096A configuration. The data show that the $\mathrm{Si}-\mathrm{O}$ and $\mathrm{O}-\mathrm{O}$ nearest-neighbour interatomic distances vary only weakly with pressure, but that the variation of the closest Si-Si distance closely mirrors the linear dimensions of the sample (and shows the same curvature that leads 

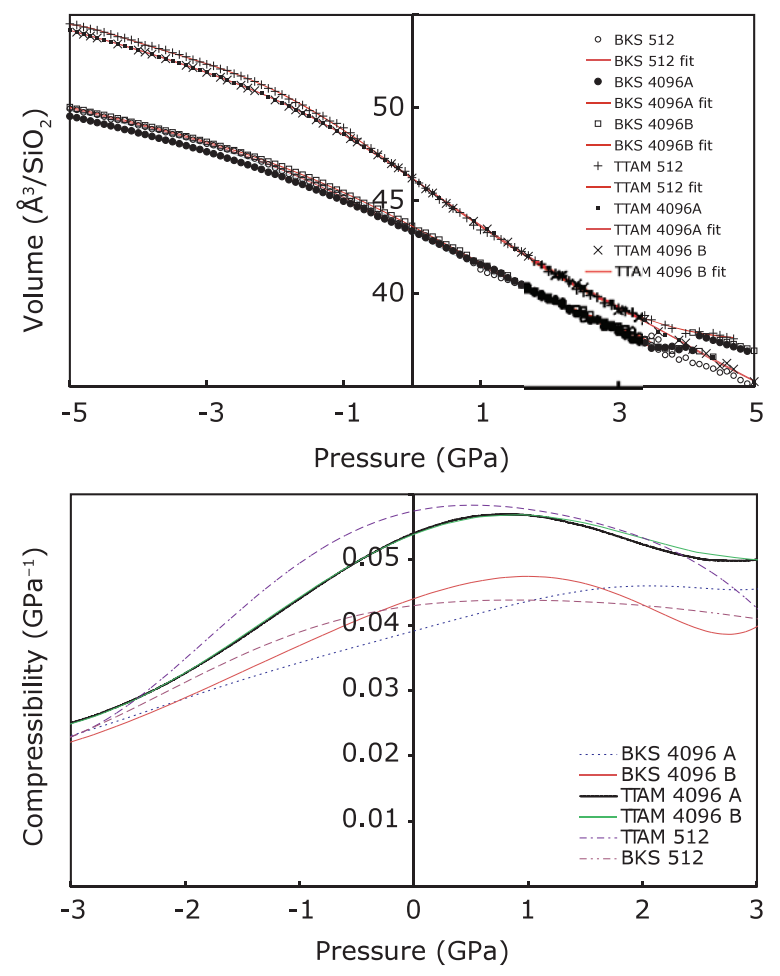

Figure 2. Simulated volume versus pressure curves for the various silica models described in this paper (top), together with the compressibility curves obtained by differentiation of the fitted volume curves (bottom).

(This figure is in colour only in the electronic version)

to a compressibility maximum). The comparison of the different data sets in figure 3 (top) suggests that the volume changes are mostly accommodated by buckling of the network, with the $\mathrm{Si}-\mathrm{Si}$ nearest-neighbour distance being the signature. Stretching of the $\mathrm{SiO}_{4}$ tetrahedra are seen in the data for $\mathrm{Si}-\mathrm{O}$ and $\mathrm{O}-\mathrm{O}$ distances at larger negative pressures, as per the idealization sketched in figure 1(a). Figure 3 (bottom) compares the variances of the same distances. The $\mathrm{Si}-\mathrm{O}$ distribution has the tightest distribution, but broadens on changing pressure towards the positive and negative pressure extremes. The $\mathrm{O}-\mathrm{O}$ distribution is much broader, but like the $\mathrm{Si}-\mathrm{O}$ distribution it is tighter in the intermediate pressure regime. The $\mathrm{O}-\mathrm{O}$ distribution at high pressure broadens considerably, reflecting the onset of $\mathrm{SiO}_{4}$ deformations with increased compaction (see figure 1(c)). Similarly, as the tetrahedra are stretched at negative pressures, this stretching is seen in as broadening of the $\mathrm{O}-\mathrm{O}$ distribution. At the same time, figure 3 (bottom) shows that the mean $\mathrm{O}-\mathrm{O}$ distance does not vary any more than the mean $\mathrm{Si}-\mathrm{O}$ distance, which together with the breadth of the distribution is consistent with the interpretation that most of the deformation of the $\mathrm{SiO}_{4}$ tetrahedra is in flexing of the $\mathrm{O}-\mathrm{Si}-\mathrm{O}$ angles. This would preserve the mean $\mathrm{O}-\mathrm{O}$ separation when averaged over the six distances within each tetrahedra, but cause broadening of the $\mathrm{O}-\mathrm{O}$ distribution. The $\mathrm{Si}-\mathrm{Si}$ distribution is tighter at low pressure and broadens on increasing pressure in a manner similar to the changes in the mean $\mathrm{Si}-\mathrm{Si}$ distance. The broadening of the distribution is consistent with the inhomogeneous buckling of the network under compaction. 

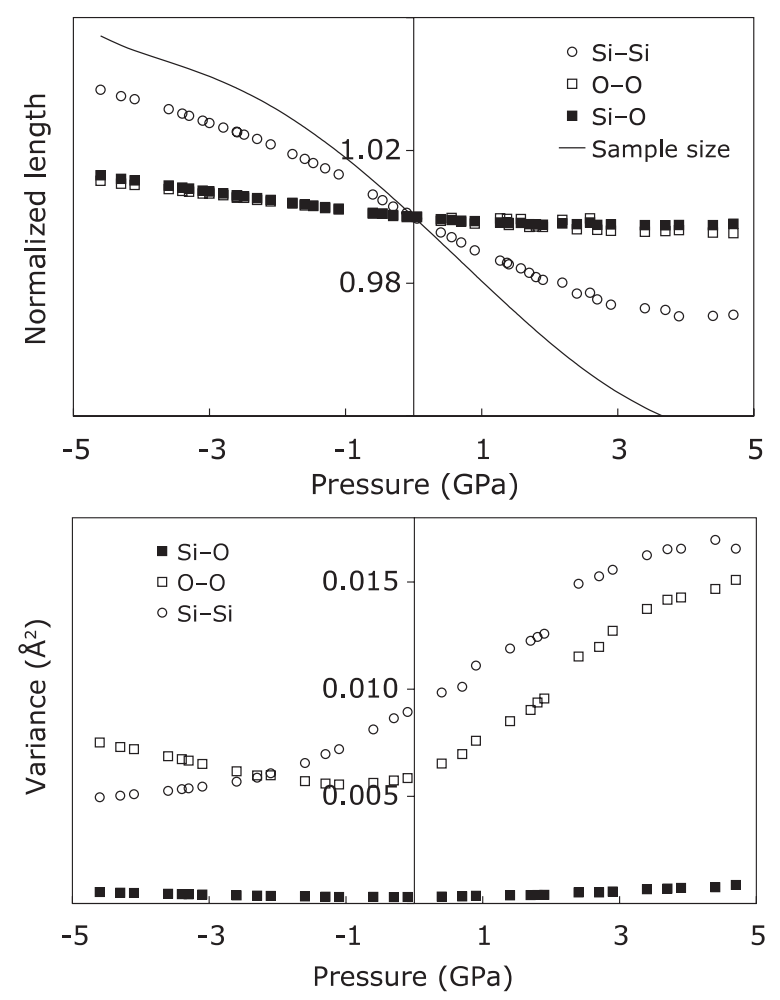

Figure 3. Top: comparison of the linear sample dimension $\left(V^{1 / 3}\right)$ and mean nearest-neighbour $\mathrm{Si}-\mathrm{O}, \mathrm{O}-\mathrm{O}$ and $\mathrm{Si}-\mathrm{Si}$ distances, all normalized to unity at $P=0$. Bottom: variances on the distributions of nearest-neighbour $\mathrm{Si}-\mathrm{O}, \mathrm{O}-\mathrm{O}$ and $\mathrm{Si}-\mathrm{Si}$ distances. Both plots are from the simulations of the 4096A configuration with the TTAM potential.

\subsection{Analysis of network flexibility}

The structural information provides information on the process of volume change, but the final part of the flexibility hypothesis that needs investigation is the supposition that the network structure is more flexible at intermediate pressures. We quantify this by comparing around 50 snapshot configurations at each pressure, and using a tool based on the mathematical technique of geometric algebra [24-26] to partition the atomic displacements into rigid unit motions (i.e. displacements and rotations of $\mathrm{SiO}_{4}$ tetrahedra) and deformations of the $\mathrm{SiO}_{4}$ tetrahedra (bond bending and stretching). For each comparison we compute the atomic displacements that would generate one configuration from the other, and then partition these into the mean square tetrahedral rotations, displacements and deformations. We then form histograms of these mean square values for all comparisons. The results for the tetrahedral rotations are shown in figure 4. The histograms show two types of dynamics: the fast vibrational motions, which are represented by the peaks in the histograms that stretch across the range of pressures, and slow/rare localized large-amplitude rotations of groups of tetrahedra, which are shown as the long high-value tails in some of the histograms. Because these latter events are rare, they limit the statistical smoothness of the plots in figure 4 . The clear result from figure 4 is that the amplitudes of the fast rotational thermal motions of the $\mathrm{SiO}_{4}$ tetrahedra are larger for intermediate pressures. This quantity acts as a proxy for the flexibility of the network, because the flexibility available for thermal fluctuations is also the flexibility available for 


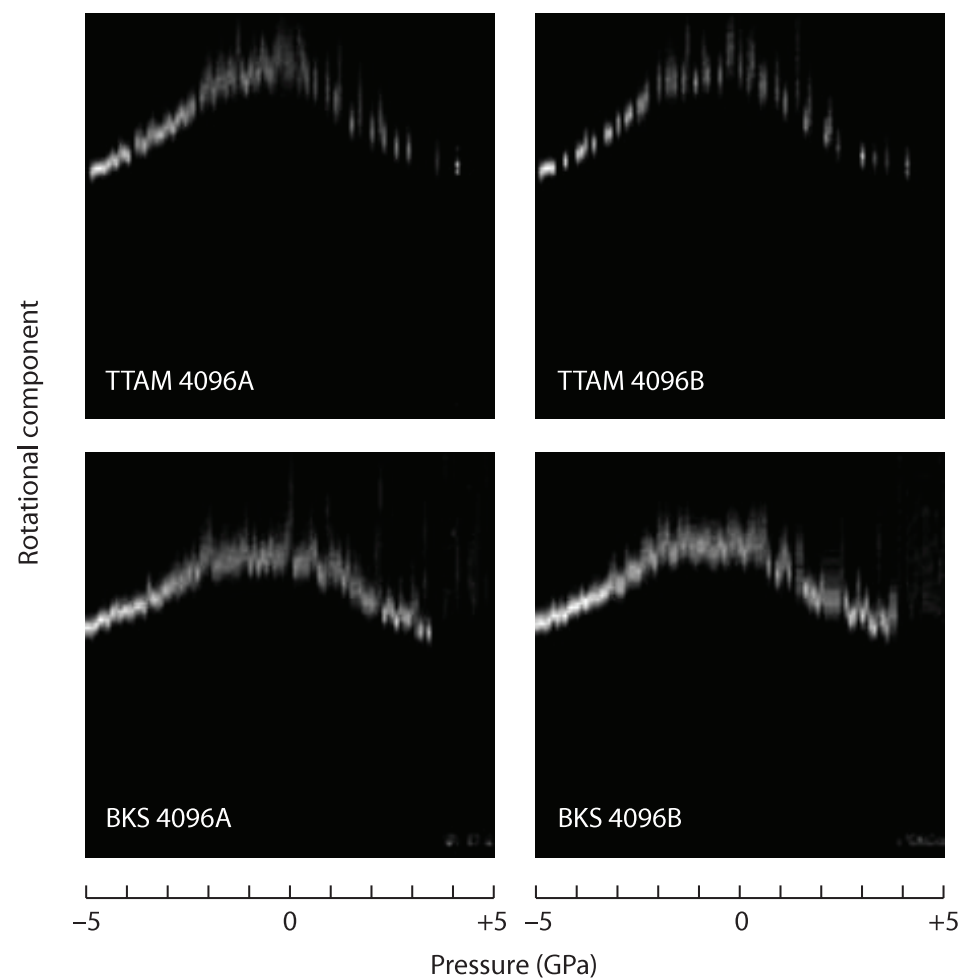

Figure 4. Four composites of histograms of the distribution of mean square rotational components of the instantaneous atomic displacements for two configurations of 4096 formula units of silica (A and B) and the two interatomic potential models (TTAM and BKS). There is a separate histogram for each pressure, which is displayed vertically, and the histograms are stacked left to right in each composite. In this representation, histogram values are indicated using a grey scale, with larger values of the histograms as white and zero values as black. Missing histograms are seen as vertical blocks of black. The key result is that the line of white, corresponding to the degree of flexibility, peaks at intermediate pressures, showing that the flexibility for rigid unit motions is greatest at the pressures corresponding to the compressibility maximum (compare with figure 2).

accommodation for the buckling of the network under changes of volume (as also is the rare localized event flexibility). It can be seen from figure 4 that the rotational flexibility has its maximum in the intermediate pressure range where the compressibility has its maximum, concordant with the flexibility hypothesis as the origin of the compressibility maximum. We note that the maxima obtained from the data in figure 4 do not correspond directly with the maxima in the derived compressibility curves show in figure 2 (bottom), but this may to some extent reflect the fact that locating the maxima in the compressibility curves is numerically rather subtle.

\section{Summary and discussion}

To summarize, we have proposed that the compressibility maximum in amorphous silica arises because there is a range of pressures over which volume changes can be accommodated by low-energy buckling of the network without the requirement for deformations of the $\mathrm{SiO}_{4}$ tetrahedra. Simulation studies of the changes in structure and dynamic fluctuations are fully consistent with the flexibility hypothesis and lend considerable detailed support to it. 
The flexibility hypothesis does not build upon the nature of the amorphous structure. Since we know that most network crystalline structures have the same or greater dynamic flexibility [27], we predict that compressibility maxima may also be observed in crystalline networks, provided that they do not undergo structural phase transitions under pressure. A compressibility maximum has been confirmed for some siliceous zeolites $[28,29]$. We noted above that a flexibility window has recently been noted to exist in zeolites [7]. There is one significant difference in that the flexibility we are discussing exists outside the constraints of symmetry and hence will be excited by thermal fluctuations, whereas the work on zeolites was constrained by symmetry. We also propose that the anomalous elastic softening of albite [30] has a similar origin, although the observation of a maximum in the compressibility will require further measurements at higher pressures. Another example is $\mathrm{ZrW}_{2} \mathrm{O}_{8}$ [31], which also has elastic softening on increasing pressure, but a maximum in compressibility will be hidden by the phase transition at $0.5 \mathrm{GPa}$. Given that $\mathrm{ZrW}_{2} \mathrm{O}_{8}$ contains a significant number of RUMs [32, 33], the flexibility hypothesis is a natural explanation for the elastic softening. The authors of [31] present a quantitative argument based on the existence of network flexibility which we believe may correspond to the low-pressure regime of our model. Other network structures such as quartz and cristobalite may not show the same effect, at least at ambient pressure, because of the effects of the phase transitions at high temperature. In such cases, the phase transition provides a dominant compression mechanism through the way that the structure can change volume via the deformation that defines the phase transition.

\section{Acknowledgments}

We are grateful to NERC and EPSRC for funding this research. The authors would like to acknowledge the use of the UK National Grid Service, North-West Grid, CamGrid and the eMinerals Minigrid in carrying out this work.

\section{References}

[1] Tsiok O B, Brazhkin V V, Lyapin A G and Khvostantsev L G 1998 Logarithmic kinetics of the amorphousamorphous transformations in $\mathrm{SiO}_{2}$ and $\mathrm{GeO}_{2}$ glasses under high-pressure Phys. Rev. Lett. 80 999-1002

[2] Trachenko K and Dove M T 2002 Densification of silica glass under pressure J. Phys.: Condens. Matter $147449-59$

[3] Trachenko K and Dove M T 2002 Floppy modes in silica glass under pressure J. Phys.: Condens. Matter $141143-52$

[4] Trachenko K and Dove M T 2003 Compressibility, kinetics, and phase transition in pressurized amorphous silica Phys. Rev. B 67064107

[5] Trachenko K, Dove M T, Hammonds K D, Harris M J and Heine V 1998 Low energy dynamics and tunneling states in silica glass Phys. Rev. Lett. $813431-4$

[6] Trachenko K O, Dove M T, Harris M J and Heine V 2000 Dynamics of silica glass: two-level tunnelling states and low-energy floppy modes J. Phys.: Condens. Matter 12 8041-64

[7] Sartbaeva A, Wells S A, Tracey M M and Thorpe M F 2006 The flexibility window in zeolites Nat. Mater. $5962-5$

[8] Huang L P and Kieffer J 2004 Amorphous-amorphous transitions in silica glass. I. Reversible transitions and thermomechanical anomalies Phys. Rev. B 69224203

[9] Huang L P, Duffrene L and Kieffer J 2004 Structural transitions in silica glass: thermo-mechanical anomalies and polyamorphism J. Non-Cryst. Solids 349 1-9

[10] Vukcevich M R 1972 A new interpretation of the anomalous properties of vitreous silica J. Non-Cryst. Solids $1125-63$

[11] Wooten F, Winer K and Weaire D 1985 Computer generation of structural models of amorphous Si and Ge Phys. Rev. Lett. 54 1392-5 
[12] Wooten F and Weaire D 1987 Modeling tetrahedrally bonded random networks by computer Solid State Phys. 40 $1-42$

[13] Tsuneyuki S, Tsukada M, Aoki H and Matsui Y 1988 1st principles interatomic potential of silica applied to molecular dynamics Phys. Rev. Lett. 61 869-72

[14] van Beest B W H, Kramer G J and van Santen R W 1990 Force fields for silicas and aluminophosphates based on ab initio calculations Phys. Rev. Lett. 64 1955-8

[15] Todorov I T and Smith W 2004 DL_POLY_3: the CCP5 national UK code for molecular-dynamics simulations Phil. Trans. R. Soc. A 362 1835-52

[16] Todorov I T, Smith W, Trachenko K and Dove M T 2006 DL_POLY_3: new dimensions in molecular dynamics simulations via massive parallelism J. Mater. Chem. 16 1911-8

[17] Allen M P and Tildesley D J 1989 Computer Simulation of Liquids (Oxford: Clarendon Press)

[18] Berendsen H J C, Postma J P M, van Gunsteren W, DiNola A and Haak J R 1984 J. Chem. Phys. 81 3684-90

[19] Bruin R P, White T O H, Walker A M, Austen K F, Dove M T, Tyer R P, Couch P A, Todorov I T and Blanchard M O 2006 Job submission to grid computing environments Proc. UK e-Science All Hands Mtg 2006 pp 754-61 (ISBN 0-9553988-0-0)

[20] Tyer R P, Couch P A, Kleese van Dam K, Todorov I T, Bruin R P, White T O H, Walker A M, Austen K F, Dove M T and Blanchard M O 2006 Automatic metadata capture and grid computing Proc. UK e-Science All Hands Mtg 2006 pp 381-4 (ISBN 0-9553988-0-0)

[21] Walker A M, Dove M T, Sullivan L A, Trachenko K, Bruin R P, White T O H, Murray-Rust P, Todorov I T, Tyer R P, Couch P A, Todorov I T, Smith W and van Dam Kleese K 2006 Anatomy of a grid-enabled molecular simulation study: the compressibility of amorphous silica Proc. UK e-Science All Hands Mtg 2006 pp 653-60 (ISBN 0-9553988-0-0)

[22] Dove M T, Sullivan L A, Walker A M, Bruin R P, White T O H, Trachenko K, Murray-Rust P, Todorov I T, Tyer R P, Couch P A, van Dam K K and Smith W 2006 Molecular dynamics in a grid computing environment: experiences using DL_POLY_3 within the eMinerals escience project Mol. Simul. 32 945-52

[23] White T O H, Murray-Rust P, Couch P A, Tyer R P, Bruin R P, Todorov I T, Wilson D J, Dove M T, Austen K F and Parker S C 2006 Application and uses of CML within the eMinerals project Proc. UK e-Science All Hands Mtg 2006 pp 606-13 (ISBN 0-9553988-0-0)

[24] Wells S A, Dove M T and Tucker M G 2002 Finding best-fit polyhedral rotations with geometric algebra J. Phys.: Condens. Matter 14 4567-84

[25] Wells S A, Dove M T, Tucker M G and Trachenko K O 2002 Real-space rigid unit mode analysis of dynamic disorder in quartz, cristobalite and amorphous silica J. Phys.: Condens. Matter 14 4645-57

[26] Wells S A, Dove M T and Tucker M G 2004 Reverse Monte Carlo with geometric analysis-RMC+GA J. Appl. Crystallogr. 37 536-44

[27] Hammonds K D, Dove M T, Giddy A P, Heine V and Winkler B 1996 Rigid-unit phonon modes and structural phase transitions in framework silicates Am. Mineral. 81 1057-79

[28] Hriljac J A 2006 High-pressure synchrotron X-ray powder diffraction studies of zeolites Crystallogr. Rev. 12 181-93

[29] Hriljac J A 2006 personal communication

[30] Benusa M D, Angel R J and Ross N L 2005 Compression of albite, NaAlSi $\mathrm{O}_{8}$ Am. Mineral. 90 1115-20

[31] Pantea C, Migliori A, Littlewood P B, Zhao Y, Ledbetter H, Lashley J C, Kimura T, Van Duijn J and Kowach G R 2006 Pressure-induced elastic softening of monocrystalline zirconium tungstate at $300 \mathrm{~K}$ Phys. Rev. B 73214118

[32] Pryde A K A, Hammonds K D, Dove M T, Heine V, Gale J D and Warren M C 1996 Origin of the negative thermal expansion in $\mathrm{ZrW}_{2} \mathrm{O}_{8}$ and $\mathrm{ZrV}_{2} \mathrm{O}_{7}$ J. Phys.: Condens. Matter 8 10973-82

[33] Tucker M G, Goodwin A L, Dove M T, Keen D A, Wells S A and Evans J S O 2005 Negative thermal expansion in $\mathrm{ZrW}_{2} \mathrm{O}_{8}$ : mechanisms, rigid unit modes, and neutron total scattering Phys. Rev. Lett. 95255501 